ACCEPTED MANUSCRIPT

\title{
Measuring the nonlocality of different types of Majorana bound states in a topological superconducting wire
}

To cite this article before publication: Chongdan Ren et al $2018 \mathrm{~J}$. Phys.: Condens. Matter in press https://doi.org/10.1088/1361-648X/aaf149

\section{Manuscript version: Accepted Manuscript}

Accepted Manuscript is "the version of the article accepted for publication including all changes made as a result of the peer review process, and which may also include the addition to the article by IOP Publishing of a header, an article ID, a cover sheet and/or an 'Accepted

Manuscript' watermark, but excluding any other editing, typesetting or other changes made by IOP Publishing and/or its licensors"

This Accepted Manuscript is @ 2018 IOP Publishing Ltd.

During the embargo period (the 12 month period from the publication of the Version of Record of this article), the Accepted Manuscript is fully protected by copyright and cannot be reused or reposted elsewhere.

As the Version of Record of this article is going to be / has been published on a subscription basis, this Accepted Manuscript is available for reuse under a CC BY-NC-ND 3.0 licence after the 12 month embargo period.

After the embargo period, everyone is permitted to use copy and redistribute this article for non-commercial purposes only, provided that they adhere to all the terms of the licence https://creativecommons.org/licences/by-nc-nd/3.0

Although reasonable endeavours have been taken to obtain all necessary permissions from third parties to include their copyrighted content within this article, their full citation and copyright line may not be present in this Accepted Manuscript version. Before using any content from this article, please refer to the Version of Record on IOPscience once published for full citation and copyright details, as permissions will likely be required. All third party content is fully copyright protected, unless specifically stated otherwise in the figure caption in the Version of Record.

View the article online for updates and enhancements. 


\title{
Measuring the nonlocality of different types of Majorana bound states in a topological superconducting wire
}

\author{
Chongdan Ren ${ }^{1}$, Ya Wu${ }^{1}$, Minglei $\mathrm{Sun}^{2}$, Sake Wang ${ }^{3}$, Hongyu \\ $\operatorname{Tian}^{4}$ \\ Department of Physics, Zunyi Normal University, Kweichow 563002, China \\ Physical Science and Engineering Division(PSE), King Abdullah University of \\ Science and Technology (KAUST), Thuwal, 23955-6900, Saudi Arabia \\ School of Science, Jinling Institute of Technology, Nanjing 211169, China \\ School of Physics and Electronic Engineering, Linyi University, Linyi 276005, China \\ E-mail: tianhongyu@lyu.edu.cn \\ 5 November 2018
}

\begin{abstract}
According to the degree of topological protection, Majorana bound states (MBSs) can be divided into three types: ideal zero-energy MBSs (IZMs), finite-energy MBSs (FEMs) and zero-energy MBSs at parity crossing points (PZMs). Herein, we investigate the nonlocality of these three types of MBSs by comparing the conductance spectra of a normal lead-topological superconducting wire-normal lead (NSN) junction and an NS junction. We find that for the FEM-related tunnelling process, the decrease in the nonlocal processes is trivially accompanied by an increase in the local processes, whereas for the IZM-related tunnelling process, the left and right tunnelling processes are completely independent. Remarkably, PZMs induce a nonlocal electron-blocking effect in which incoming electrons from the left lead cannot participate in local Andreev reflection unless the right lead is present, even though no nonlocal tunnelling processes occur in the right lead of an NSN junction. We show that this PZM-mediated nonlocal electron-blocking effect is due to the nonlocal coupling of the left lead to the more distant PZM and that the phase difference between the two end PZMs is $\pi / 2$. Our findings provide an experimentally accessible method for characterizing MBSs by probing their different nonlocal signatures.
\end{abstract}

PACS numbers: 74.50.+r, 74.20.Rp, 71.70.Ej, 74.25.F-, 72.25.-b, 85.25.Cp

Keywords: topological superconductor, Majorana bound state, nonlocality

Submitted to: J. Phys.: Condens. Matter

\section{Introduction}

Majorana bound states (MBSs) are exotic self-conjugate Bogoliubov quasiparticles that appear as zero-energy end states in topological superconductors [1, 2]. Due to 
the unique non-Abelian statistics of MBSs, they provide an ideal platform for faulttolerant topological quantum computing $[3,4]$. Various routes are available for realizing topological superconductivity $[5,6,7,8,9,10,11,12,13]$. Among such routes, a spinorbit-coupled semiconducting nanowire with proximity-induced superconductivity that is subject to a magnetic field $[13,12]$ (a system that is henceforth referred to as an "S-nanowire" for simplicity) has been identified as the most feasible platform due to its straightforward nanofabrication. Theory predicts that above a certain critical magnetic field, i.e., at $B>B_{c}$, two spatially separated MBSs emerge at the endpoints of an S-nanowire [13]. This separation, or nonlocality, is the key to the topological protection of the MBSs, which makes MBS-based qubits immune to decoherence caused by local disturbances.

According to the degree of topological protection, MBSs can be classified into three types: ideal zero-energy MBSs (IZMs), finite-energy MBSs (FEMs) and zero-energy MBSs at parity crossing points (PZMs) [14]. In a sufficiently long S-nanowire, the two end MBSs do not overlap and are degenerate zero-energy states (IZMs). In a realistic S-nanowire of finite length $L$, the two end MBSs overlap to some extent and become finite-energy states $[15,16,17]$ (i.e., FEMs), $E_{m} \approx e^{-L / l_{M}} \cos \left(k_{F} L\right)$, where $l_{M}$ is the spatial extension of MBSs and $k_{F}$ is the effective Fermi wave-vector. Moreover, the FEMs can be either empty or occupied, which are characterized by an even or odd parity state, and the energies of even and odd parity FEMs are oscillatory with some external parameters, such as magnetic field, chemical potential, or length [15]. At parity crossing points (i.e., $k_{F} L=\pi / 2$ ), the two parity states cross each other at zero energy and the MBSs become zero-energy states again, i.e., PZMs. Recently, a topological quality factor is introduced to characterize the degree of nonlocality of MBSs $[14,18]$, and it is found that PZMs is less topologically protected than IZMs even though both of them are zero-energy states.

Different types of paired MBSs may exhibit unique nonlocal properties; thus, the search for the nonlocal effects induced in paired MBSs can provide important signatures for the existence of MBSs. Various schemes have been developed for manifesting the nonlocality of MBSs. Early proposals required a nonlocal measurement of the system, such as crossed Andreev reflection (AR), in which two electrons from different ends combine to form a Cooper pair assisted by FEMs [19, 20, 21, 22, 23, 24, 25], or a teleportation-like process involving the transfer of an electron between well-separated points with the aid of IZMs $[26,27,28]$. Recently, a structure consisting of a quantum dot coupled to the S-nanowire has received much attention. As an alternative to manipulating and probing the MBSs [29, 30] or the detection of a topological phase transition [31], hybrid S-nanowire-quantum dot devices can serve as a simple means of demonstrating the nonlocality of MBSs by local probing the differential conductance in a homogeneous [14, 18, 32] or inhomogeneous [33] S-nanowire or the Fano resonances [34]. In addition, the nonlocality and delocalization of MBSs have also been investigated in a system of mixed dimensionality [35]. However, the above studies mainly focused on the nonlocal transport properties of FEMs and IZMs; by contrast, the unique nonlocal 
transport mediated by PZMs has received less attention. In the recent work [24], we reported a PZMs-induced spin blocking effect in which the one side transport processes are nonlocally influenced by the spin direction of the other side PZM. However,this scheme is too complicated and thus pose experimental difficulties. Therefore, it is desirable to propose an experimentally accessible approach to illustrate the nonlocality of PZMs.

In this paper, we investigate the nonlocal transport properties of all three types of MBSs, especially PZMs, in a normal lead-topological superconducting wire-normal lead (NSN) junction and in an NS junction, as shown in Fig. 1(a) and (b). Such NSN and NS structures have been extensively studied [19, 36, 20, 37, 38, 39, 30, 40]. However, most previous works have focused on the nonlocality of FEMs and the zeroenergy conductance peaks related to IZMs. Very little research has been conducted on the nonlocal transport properties of PZMs. We find that, for the transport process mediated by FEMs, both local AR and nonlocal crossed Andreev reflection (CAR) as well as elastic cotunnelling (ET) processes exist in the NSN junction and the local AR is trivially enhanced in the NS junction (as shown in Fig. 1(c) and (d)), whereas for the transport process mediated by IZMs, the presence or absence of the right lead does not affect the transport behaviour in the left lead (as shown in Fig. 1(g) and (h)). Notably, for the transport process mediated by PZMs, only local AR exists in the NSN junction, similar to the IZM case, but the local AR disappears in the NS junction even though no tunnelling processes occur in the right lead of the NSN junction (as shown in Fig. 1(e) and (f)). We refer to this new phenomenon as the PZM-mediated nonlocal electron-blocking effect. We further investigate the underlying mechanisms that drive the different transport behaviours for the three types of MBSs, and we find that these different transport behaviours are directly related to the nonlocal coupling between the electron at one end and the more distant MBS at the other end. Because of the presence (absence) of this nonlocal coupling, the right lead will affect (not affect) the tunnelling process on the left side for the FEM- and PZM (IZM)-related transport processes. These differences in the transport properties provide some insights regarding the nonlocality of MBSs.

The remainder of this work is organized as follows. In Sec. II, the system Hamiltonian and the theoretical formula for calculating the differential conductance are presented. In Sec. III, we present the numerical results and a discussion. A brief conclusion is given in the last section.

\section{Model and formalism}

The proposed NSN structure, in which the two MBSs emerging at the endpoints of the S-nanowire are tunnel coupled to two normal leads, is schematically illustrated in Fig. 1(a). The tight-binding Hamiltonian describing the system is given by

$$
H=\sum_{i, \sigma} c_{i, \sigma}^{\dagger}(t-\mu) \sigma_{0} c_{i, \sigma}
$$




$$
\begin{aligned}
& +\sum_{i \in T S, \sigma} c_{i, \sigma}^{\dagger} B \sigma_{z} c_{i, \sigma} \\
& -\frac{1}{2} \sum_{\langle i, j\rangle, \sigma}\left(t_{i j} c_{i \sigma}^{\dagger} \sigma_{0} c_{j, \sigma}+\text { h.c. }\right) \\
& -\frac{1}{2} \sum_{\langle i, j\rangle \in T S, \sigma \sigma^{\prime}}\left(t_{s o} c_{i \sigma}^{\dagger}\left(i \sigma_{y}\right) c_{j, \sigma^{\prime}}+\text { h.c. }\right) \\
& +\sum_{i \in T S}\left(\Delta c_{i, \uparrow} c_{i, \downarrow}+\text { h.c. }\right)
\end{aligned}
$$

where $c_{i, \sigma}^{\dagger}$ is a creation operator for an electron of spin $\sigma$ at site $i$, the symbol $\langle i, j\rangle$ denotes nearest-neighbour sites $i$ and $j, \mu$ is the chemical potential, $B$ is the Zeeman energy along the $z$-direction, $t_{s o}$ is the spin-orbit coupling strength and $\Delta$ is the superconducting pairing amplitude. Here, the system is divided into three parts: the left and right nanowire subsystems for $i<0$ and $i>l$, respectively, and the S-nanowire in between. The hopping amplitude is assumed to be the same everywhere, $t_{i j}=t$, except for the coupling between the N-nanowire and S-nanowire, for which $t_{i j}=t_{L(R)}$. The NS junction in Fig. 1(b) is realized by setting $t_{R}=0$.

To investigate the nonlocal transport properties, the scattering matrix is employed to calculate the differential conductance. With the help of the recursive Green's function method, the scattering matrix can be written as $[22,20,25,23,24]$

$$
S_{i j}^{\alpha \beta}=-\delta_{i j} \delta_{\alpha \beta}+i\left[\Gamma^{1 / 2}\right]_{i}^{\alpha} \cdot\left[G^{r}\right]_{\alpha \beta}^{i j} \cdot\left[\Gamma^{1 / 2}\right]_{j}^{\beta}
$$

Here, $S_{i j}^{\alpha \beta}$ is an element of the scattering matrix that indicates the scattering amplitude of an incoming $\beta$ particle from the $j$ th lead to be scattered as particle $\alpha$ to the $i$ th lead, where $i$ and $j$ denote the left and right leads, respectively, and $\alpha$ and $\beta$ represent the electron and hole, respectively. $G^{r}=\left[E-H_{T S}-\sum_{l, \alpha}\left(\Sigma_{l}^{\alpha}\right)^{r}\right]^{-1}$ is the retarded Green's function of the model. $\Gamma_{i}^{\alpha}=i\left[\left(\Sigma_{i}^{\alpha}\right)^{r}-\left(\Sigma_{i}^{\alpha}\right)^{\alpha}\right]$ is the broadening function and $\left(\Sigma_{i}^{\alpha}\right)^{r(a)}$ is the retarded (advanced) self-energy of the $\alpha$ particle in the $i$ th lead, which can be calculated by the recursive iteration method $[41,42,43,44]$.

With the scattering matrix, the differential conductance of the $i$ th lead can be calculated as $[25,20,23,24]$

$$
G_{i}=\frac{e^{2}}{h} \operatorname{Tr}\left[I-r_{i i}^{e e}-r_{i j}^{e e}+r_{i i}^{h h}+r_{i j}^{h h}\right]
$$

with $r_{i j}^{x y}(E)=S_{i j}^{x e}(E)\left[S_{i j}^{y e}(E)\right]^{\dagger}(x, y \in\{e, h\})$. The physical meaning of the scattering matrix is clear: $r_{i i}^{e e}$ and $r_{i i}^{h h}$ are the local normal reflection (NR) and local AR coefficients in the $i$ th lead, respectively, and $r_{i j}^{e e}$ and $r_{i j}^{h h}$ are the nonlocal ET and CAR coefficients of electrons from the $i$ th lead to the $j$ th lead, respectively. Here, the CAR (ET) process is related to the injection of an electron into one MBS followed by the emission of a hole (electron) by the other MBS, which is a direct probe of the nonlocality of the MBSs $[19,20,39]$. In an NSN junction, both local and nonlocal processes may contribute to the conductance, whereas in an NS junction, only local processes exist. 


\section{Results and discussion}

Using the above model and formula, the nonlocal transport properties of different types of MBSs are investigated. In the numerical calculations, we use a set of parameters that are consistent with the experiments in Ref. [45]. We set the hopping energy $t$ as the unit energy $t=1$. The other parameters are set to $t_{s o}=0.1 t, \Delta=0.04 t$ and $B=0.08 t$. The chemical potential $\mu$ is chosen to lie in the range from $-0.04 t$ to $0.06 t$ such that the S-nanowire is in the topological nontrivial phase, i.e., $B>\sqrt{\mu^{2}+\Delta^{2}}$. The couplings between the N-nanowires and the S-nanowire in the NSN junction and in the NS junction are set to $t_{L}=t_{R}=0.1 t$ and $t_{L}=0.1 t, t_{R}=0$, respectively.

Before presenting the results of the differential conductance $G_{L}$ of the left lead in the NSN and NS junctions as a function of chemical potential $\mu$ and incident energy $E$, it is informative to first discuss the variation of conductance $G_{L}$ in an NSN junction with the length of nanowires $L$ and incident energy $E$ as shown in Fig. 2. The conductance spectra display sharp peaks of large amplitude (light colour) when the incident energy equals the coupling energy of MBSs $\left(E=E_{M}\right)[20,23,24,25]$; therefore, the MBSs can manifest themselves from the conductance peaks. It can be seen that $G_{L}$ exhibits an oscillatory decaying behavior and crosses the zero-energy points as the length of the wire, $L$, is increased. For long enough wires, the amplitude of the oscillations is reduced and even totally negligible, where the conductance spectra become zero energy peaks. Therefore, the MBSs in the endpoints of a relatively short S-nanowire are FEMs or PZMs, and the MBSs at the endpoints of a long enough S-nanowire are IZMs. In the discussion below, the length of a short S-nanowire that hosts FEMs and PZMs is set to $L=40 a$, while the length of a long S-nanowire that hosts IZMs is set to $L=200 a$.

In Fig. 3(a), the differential conductance $G_{L}$ of the left lead in an NSN junction with a short S-nanowire is plotted as a function of chemical potential $\mu$ and incident energy $E$. The conductance peaks $G_{L}$ oscillates and crosses the zero-energy points as the chemical potential varies. Note that except for the apparent conductance peaks with quantized height $e^{2} / h$ related to the even and odd parity FEMs, there are some isolated points at zero energy with $G_{L}=2 e^{2} / h$ that directly arise from scattering process with the aid of PZMs. To demonstrate the nonlocality of FEMs and PZMs, the contributions of conductance)(AR, CAR and ET) are presented in Fig. 3(b), (c) and (d). As shown, both a local AR process and nonlocal CAR and ET processes exist for the conductance spectra related to FEMs, whereas only a local AR process contributes to PEM-related conductance spectra (as shown in Fig. 1(c), (d)). Moreover, the ET process displays an even-odd parity interference effect, whereas the CAR process does not, which is directly from the self-Hermitian property of MBSs[25]. From the above results, it may be hypothesized that the left and right tunnelling processes mediated by PZMs are completely independent and have no correlation, while the cross-correlation arises through the two spatially separated leads for the transport processes mediated by FEMs. To verify this hypothesis, we would like to compare the difference of conductance in an NS junction with that in an NSN junction to explore the influence of nonlocal 
processes on the local process.

In Fig. 3(e), the $G_{L}$ of the normal lead in a two-terminal NS junction with a short S-nanowire is plotted as a function of $\mu$ and $E$. At first glance, one observes that the conductance peaks mediated by FEMs are quantized as $G_{L}=2 e^{2} / h$ (as shown in Fig. 3(e)). This result is not surprising due to the disappearance of the nonlocal CAR and ET processes, and only the resonance AR process contributes to the conductance. A more careful examination, however, reveals that the conductance peaks mediated by PZMs are no longer $2 e^{2} / h$ but rather 0 , as shown in the inset of Fig. 3(b), which is different from the case of a three-terminal NSN junction. This result means that an electron from the left lead cannot participate in the local AR unless the right lead is present, even though no nonlocal processes occur in the right lead of an NSN junction. This PZM-induced nonlocal electron-blocking effect has never been reported before even though these NSN and NS junctions have been extensively investigated. Although previous studies have also reported similar results $\left(G_{L}=0\right.$ at $\left.V=0\right)[46,47,48]$, they are actually not the same as ours. In fact, their results are similar to the FEM-induced conductance in the NS junction shown above, where $G_{L}=0$ at $V=0$ and $G_{L}=2 e^{2} / h$ at the coupling energy of the MBSs, while the PZM-induced conductance is zero when the incident energy is equal to the coupling energy of the PZMs.

Finally, we consider the IZM-induced transport feature in a long S-nanowire. Fig. 4(a) refers to the NSN junction, while Fig. 4(e) refers to the NS junction. As shown, these two conductance spectra are nearly identical. Moreover, the conductance peaks at zero energy have a height of $2 e^{2} / h$ and are nearly invariant as the chemical potential changes. Moreover, the contributions of $G_{L}$ in the NSN junction are also plotted in Fig. 4(b)-(d). Only a local AR process exists, the same as in the case of the PZM-induced conductance in an NSN junction. Moreover, the conductance spectra in the NS junction are the same as in the NSN junction, as compared between Fig. 4(a) and (e), which is different from the case of PZMs. Therefore, although IZMs and PZMs both have zero energy, they exhibit different nonlocal transport properties, which is directly related to their different degrees of topological protection.

To explain the above results, particularly the nonlocal blocking effect of PZMs, we introduce an effective Hamiltonian as

$$
\begin{aligned}
& H_{\text {eff }}=H_{L}+H_{R}+H_{S}+H_{T L}+H_{T R} \\
& H_{L(R)}=\sum_{p} \xi_{p, L(R)} c_{p}^{\dagger} c_{p} \\
& \overbrace{S}=i E_{M}^{\prime} \gamma_{1} \gamma_{2} \\
& H_{T L(T R)}=\sum_{p, i \in(1,2)}\left(t_{i, L(R)}^{*} c_{p}^{\dagger}-t_{i, L(R)} c_{p}\right) \gamma_{i}
\end{aligned}
$$

Here, $H_{L / R}$ describe the left/right normal lead, and $H_{M}$ is the Hamiltonian of two coupled MBSs, where $E_{M}$ is the coupling strength between the two end MBSs. For IZMs and PZMs, $E_{M}=0$, and for FEMs, $E_{M}>0$. The couplings between the left (right) lead and the two MBSs are described by $H_{T L}\left(H_{T R}\right)$. Note that $H_{T L(T R)}$ 
contains two terms: a local coupling term with a strength of $t_{1(2), L(R)}$ between the left (right) lead and left (right) MBS $\gamma_{1(2)}$ and a nonlocal coupling term with a strength of $t_{2(1), L(R)}$ between the left (right) lead and right (left) MBS. Moreover, the relationship between nonlocal coupling terms and local coupling terms can be given by $t_{2, L(1, R)} \propto \mathrm{e}^{\mathrm{i} k_{F} L} \mathrm{e}^{-L / l_{M}} t_{1, L(2, R)}[16,17,25]$. For PZMs, there is a $\pi / 2$ phase shift between $t_{2, L(1, R)}$ and $t_{1, L(2, R)}$ because $k_{F} L=\pi / 2$ [25]. For IZMs, the nonlocal coupling term can be neglected because $L \rightarrow \infty$.

The scattering matrix in a model-independent model can be written as $[19,21]$,

$$
S(E)=1-2 i \pi W^{\dagger}\left(E-H_{S}+\mathrm{i} \pi W W^{\dagger}\right)^{-1} W
$$

where $W$ describes the coupling between $H_{S}$ and $H_{L(R)}$. In our case, the coupling matrix $W$ is given by

$$
W=\left(\begin{array}{cccc}
t_{1 L} & t_{1 R} & t_{1 L}^{*} & t_{1 R}^{*} \\
t_{2 L} & t_{2 R} & t_{2 l}^{*} & t_{2 R}^{*}
\end{array}\right)
$$

Substituting Eq. (4) into Eq. (5) provides the electron and hole blocks of the scattering matrix,

$$
S=\left(\begin{array}{ll}
s^{e e} & s^{e h} \\
s^{h e} & s^{h h}
\end{array}\right)=\left(\begin{array}{cc}
1+A & A \\
A & 1+A
\end{array}\right)
$$

The component of scattering matrix $A=s^{\text {he }}$ describes the AR of an electron into a hole; the two diagonal terms correspond to local AR processes occurring at the left and right leads, respectively; and the off-diagonal terms correspond to nonlocal CAR processes between the two leads.

For FEMs, the nonlocal coupling terms $t_{2 L}$ and $t_{1 R}$ are far less than the coupling strength between the two end FEMs $\left(t_{2 L}\left(t_{1 R}\right) \ll E_{M}\right)$; therefore, they do not drastically affect the results and can be neglected. Then, the matrix $A$ can be expressed as

$$
A \simeq \frac{1}{Z}\left(\begin{array}{c}
i \Gamma_{1 L} E-\Gamma_{1 L} \Gamma_{2 R}-\sqrt{\Gamma_{1 L} \Gamma_{2 R}} E_{M} \\
\left.\sqrt{\Gamma_{1 L} \Gamma_{2 R}} E_{M}\right) i \Gamma_{2 R} E-\Gamma_{1 L} \Gamma_{2 R}
\end{array}\right)
$$

where $Z=E_{M}^{2}-E^{2}-i E\left(\Gamma_{1 L}+\Gamma_{2 R}\right)$ and $\Gamma_{\alpha \beta}=2 \pi\left|t_{\alpha \beta}\right|^{2}$. Therefore, for FEM-related transport processes, both local AR processes and nonlocal CAR and ET processes exist in an NSN junction. In the two-terminal NS case where $\Gamma_{2 R}=0, A$ can be reduced to $A=\left(\begin{array}{ll}1 & 0 \\ 0 & 0\end{array}\right)$. Therefore, a resonant local AR with probability of 1 occurs at the left lead that coincides with the numerical results above.

Next, we consider the PZM case $\left(E_{M}=0\right)$. Because a $\pi / 2$ phase shift occurs between $t_{2, L(1, R)}$ and $t_{1, L(2, R)}$, the matrix $A$ can be obtained as

$$
A=\frac{1}{Z}\left(\begin{array}{ll}
\mathrm{i} \Gamma_{1 L}\left(E+\mathrm{i} \Gamma_{2 R}\right)-\mathrm{i} \Gamma_{2 L}\left(E+\mathrm{i} \Gamma_{1 R}\right) & \left(\sqrt{\Gamma_{1 R} \Gamma_{2 L}}+\sqrt{\Gamma_{1 L} \Gamma_{2 R}}\right) \\
\left(\sqrt{\Gamma_{1 R} \Gamma_{2 L}}+\sqrt{\Gamma_{1 L} \Gamma_{2 R}}\right) & +E\left(\sqrt{\Gamma_{1 L} \Gamma_{1 R}}+\sqrt{\Gamma_{2 L} \Gamma_{2 R}}\right) \\
\times\left(E_{M}+\mathrm{i}\left(\sqrt{\Gamma_{1 L} \Gamma_{2 L}}+\sqrt{\Gamma_{1 R} \Gamma_{2 R}}\right)\right) & \mathrm{i} \Gamma_{2 R}\left(E+\mathrm{i} \Gamma_{1 L}\right)-\mathrm{i} \Gamma_{1 R}\left(E+\mathrm{i} \Gamma_{2 L}\right) \\
+E\left(\sqrt{\Gamma_{1 L} \Gamma_{1 R}}+\sqrt{\Gamma_{2 L} \Gamma_{2 R}}\right) &
\end{array}\right)
$$


where $Z=E_{M}^{2}-\left(E+\mathrm{i} \Gamma_{1 L}+\mathrm{i} \Gamma_{1 R}\right)\left(E+\mathrm{i} \Gamma_{2 R}+\mathrm{i} \Gamma_{2 L}\right)$. As the energy of an incoming electron equals the energy of the PZMs $\left(E=E_{M}=0\right)$, the matrix $A$ can be simplified to $A=\left(\begin{array}{ll}1 & 0 \\ 0 & 0\end{array}\right)$, which means that the nonlocal processes do not exist in an NSN junction and only a resonant local Andreev refection with a probability of 1 exists. Conversely, in the NS junction, $\Gamma_{2 R}=\Gamma_{1 R}=0$, and the matrix $A$ becomes a null matrix $A=\left(\begin{array}{ll}0 & 0 \\ 0 & 0\end{array}\right)$. This result suggests that the absence of the right lead prevents the electron from the left lead from participating in the local resonance AR process.

In contrast, the nonlocal coupling terms $t_{2 L}$ and $t_{1 R}$ do not exist for IZM-related transport. Therefore, the matrix $A$ remains the same regardless of whether the right lead is present, i.e., $A=\left(\begin{array}{ll}1 & 0 \\ 0 & 0\end{array}\right)$. This indicates that the paired IZMs are purely topological protective states that are immune to local disturbances.

\section{Conclusion}

In summary, we have investigated the nonlocal transport properties of the three types of MBSs through comparing the differences in conductance spctra between NSN and NS junctions. We have found that, for the FEM-related transport process, the local AR is trivially enhanced when the nonlocal processes are restrained, whereas for the IZMrelated transport process, the tunnelling processes in the two normal leads are mutually independent. All of these findings agree with previous investigations [20, 25, 23, 24]. However, previous research on PZM-related transport phenomena has been minimal. We reveal a striking nonlocal electron-blocking effect of PZMs in an NS junction, where the absence of the right lead prohibits the electron from the left lead from participating in the local AR. The underlying reasons have been further investigated, indicating that the different transport behaviours are directly related to the nonlocal coupling of the lead on one side to the PZM on the other side. We hope that our simple proposals can be demonstrated experimentally in the foreseeable future.

\section{Acknowledgements}

This work was supported by the National Natural Science Foundation of China, Grant Nos. 11864047 and 11704165; the Science Foundation of Guizhou Science and Technology Department, Grant No. QKHJZ[2015]2150; the Science Foundation of Guizhou Provincial Education Department, Grant No. QJHKYZ[2016]092 and the Major Research Project for Innovative Group of Education Department of Guizhou Province under Grants No. KY[2018]028. 


\section{References}

[1] Kitaev A Y 2001 Phys.Usp. 44131

[2] Read N and Green D 2000 Phys. Rev. B 6110267

[3] Kitaev A Y 2003 Ann. Phys.(N.Y.) 3032

[4] Nayak C, Simon S H, Stern A, Freedman M and Das Sarma S 2008 Rev. Mod. Phys. 801083

[5] Fu L and Kane C L 2008 Phys. Rev. Lett. 100096407

[6] Fu L and Kane C L 2009 Phys. Rev. B 79324

[7] Vazifeh M M and Franz M 2013 Phys. Rev. Lett. 111206802

[8] Nadj-Perge S, Drozdov I K, Bernevig B A and Yazdani A 2013 Phys. Rev. B 88020407

[9] Pientka F, Glazman L I and von Oppen F 2013 Phys. Rev. B 8875

[10] Sau J D, Lutchyn R M, Tewari S and Das Sarma S 2010 Phys. Rev. Lett. 104040502

[11] Alicea J 2010 Phys. Rev. B 81125318

[12] Oreg Y, Refael G and von Oppen F 2010 Phys. Rev. Lett. 105177002

[13] Lutchyn R M, Sau J D and Das Sarma S 2010 Phys. Rev. Lett. 105077001

[14] Prada E, Aguado R and San-Jose P 2017 Phys. Rev. B 96085418

[15] Das Sarma S, Sau J D and Stanescu T D 2012 Phys. Rev. B 86220506

[16] Prada E, San-Jose P and Aguado R 2012 Phys. Rev. B 86180503

[17] Rainis D, Trifunovic L, Klinovaja J and Loss D 2013 Phys. Rev. B 87024515

[18] Clarke D J 2017 Phys. Rev. B 96201109

[19] Nilsson J, Akhmerov A R and Beenakker C W J 2008 Phys. Rev. Lett. 101120403

[20] Liu J, Zhang F C and Law K T 2013 Phys. Rev. B 88064509

[21] Zocher B and Rosenow B 2013 Phys. Rev. Lett. 111036802

[22] Law K T, Lee P A and Ng T K 2009 Phys. Rev. Lett. 103237001

[23] Ren C D, Chan K S and Wang J 2014 J. Phys.: Condens. Matter 26175702

[24] Ren C D, Yang J, Xiang J, Wang S and Tian H 2017 J. Phys. Soc. Jpn. 86124715

[25] Liu J, Song J, Sun Q F and Xie X C 2017 Phys. Rev. B 96195307

[26] Fu L 2010 Phys. Rev. Lett. 104056402

[27] Wang P, Cao Y, Gong M, Li S S and Li X Q 2014 Phys. Lett. A 378937

[28] Tewari S, Zhang C, Das Sarma S, Nayak C and Lee D H 2008 Phys. Rev. Lett. 100027001

[29] Ptok A, Kobiałka A and Domański T 2017 Phys. Rev. B 96195430

[30] Stenger J P T, Woods B D, Frolov S M and Stanescu T D 2018 Phys. Rev. B 98107

[31] Chevallier D, Szumniak P, Hoffman S, Loss D and Klinovaja J 2018 Phys. Rev. B 97045404

[32] Deng M T, Vaitiekènas S, Prada E, San-Jose P, Nygård J, Krogstrup P, Aguado R and Marcus C M 2018 Phys. Rev. B 98085125

[33] Peñaranda F, Aguado R, San-Jose P and Prada E 2018 arXiv:180\%.11924

[34] Schuray A, Weithofer L and Recher P 2017 Phys. Rev. B 96085417

[35] Kobiałka A, Domański T and Ptok A 2018 arXiv:1808.05281

[36] Chevallier D, Sticlet D, Simon P and Bena C 2012 Phys. Rev. B 85944

[37] Wang P, Cao Y, Gong M, Xiong G and Li X Q 2013 EPL 10357016

[38] Lobos A M and Sarma S D 2015 New J. Phys. 17065010

[39] Liu C X, Sau J D, Stanescu T D and Das Sarma S 2017 Phys. Rev. B 96075161

[40] Cayao J, Black-Schaffer A M, Prada E and Aguado R 2018 Beilstein J. Nanotechnol. 91339

[41] Lee P A and Fisher D S 1981 Phys. Rev. Lett. 47882

[42] Lee D H and Joannopoulos J D 1981 Phys. Rev. B 234997

[43] Sancho M P L, Sancho J M L and Rubio J 1984 J. Phys F: Met. Phys. 141205

[44] Sancho M P L, Sancho J M L, Sancho J M L and Rubio J 1985 J. Phys F: Met. Phys. 15851

[45] Mourik V, Zuo K, Frolov S M, Plissard S R, Bakkers E P A M and Kouwenhoven L P 2012 Science 3361003

[46] Flensberg K 2010 Phys. Rev. B 82180516

[47] Weithofer L, Recher P and Schmidt T L 2014 Phys. Rev. B 90230 
[48] Zazunov A, Sodano P and Egger R 2013 New J. Phys. 15035033 

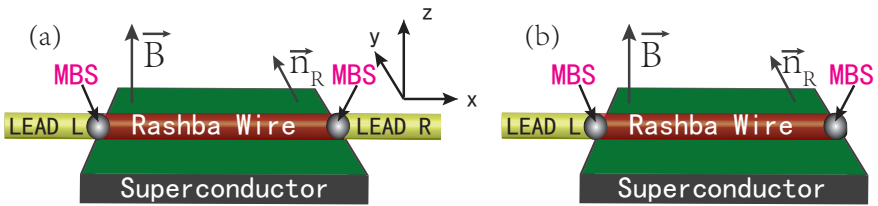

(c)

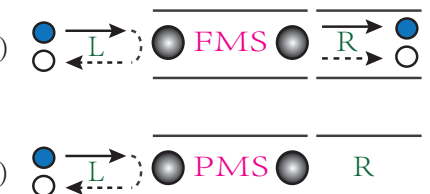

(e)

(g) $\mathrm{O} \underset{\mathrm{L}}{\longrightarrow} \mathrm{O}$ IMS $\mathrm{O}$ (d) $\mathrm{O} \underset{\& \mathrm{~L}}{\longrightarrow}: \overrightarrow{\mathrm{OFMS}}$

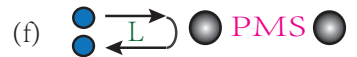

(h) $\mathrm{O} \underset{\mathrm{O}}{\mathrm{O}} \mathrm{O}$ IMS $\mathrm{O}$

Figure 1. (a) Schematic of the NSN junction, in which two normal leads are coupled to the ends of a topological superconductor that supports MBSs. The topological superconductor is realized by using a nanowire with Rashba spin-orbit coupling in proximity to a superconductor and subject to an external magnetic field $B$. The Rashba direction is denoted by $\overrightarrow{n_{R}}$. The nanowire and magnetic field are in the $x$ and $z$-directions, respectively. (b) The NS junction is realized by decoupling the right normal lead from the NSN junction. (c)-(h) show the explicit tunnelling processes mediated by three types of MBSs in an NSN junction and an NS junction. For FEMrelated transport, electrons from the left lead undergo both local AR and nonlocal CAR and ET in an NSN junction (c) and only undergo local resonant AR in an NS junction (d). For PZM-related transport, (electrons from the left lead undergo local resonant AR in an NSN junction (e) and are completely normally reflected in an NS junction (f). For IZM-related transport, the local resonant AR at the left lead is unaffected by the presence $(\mathrm{g})$ or absence $(\mathrm{h})$ of the right lead.
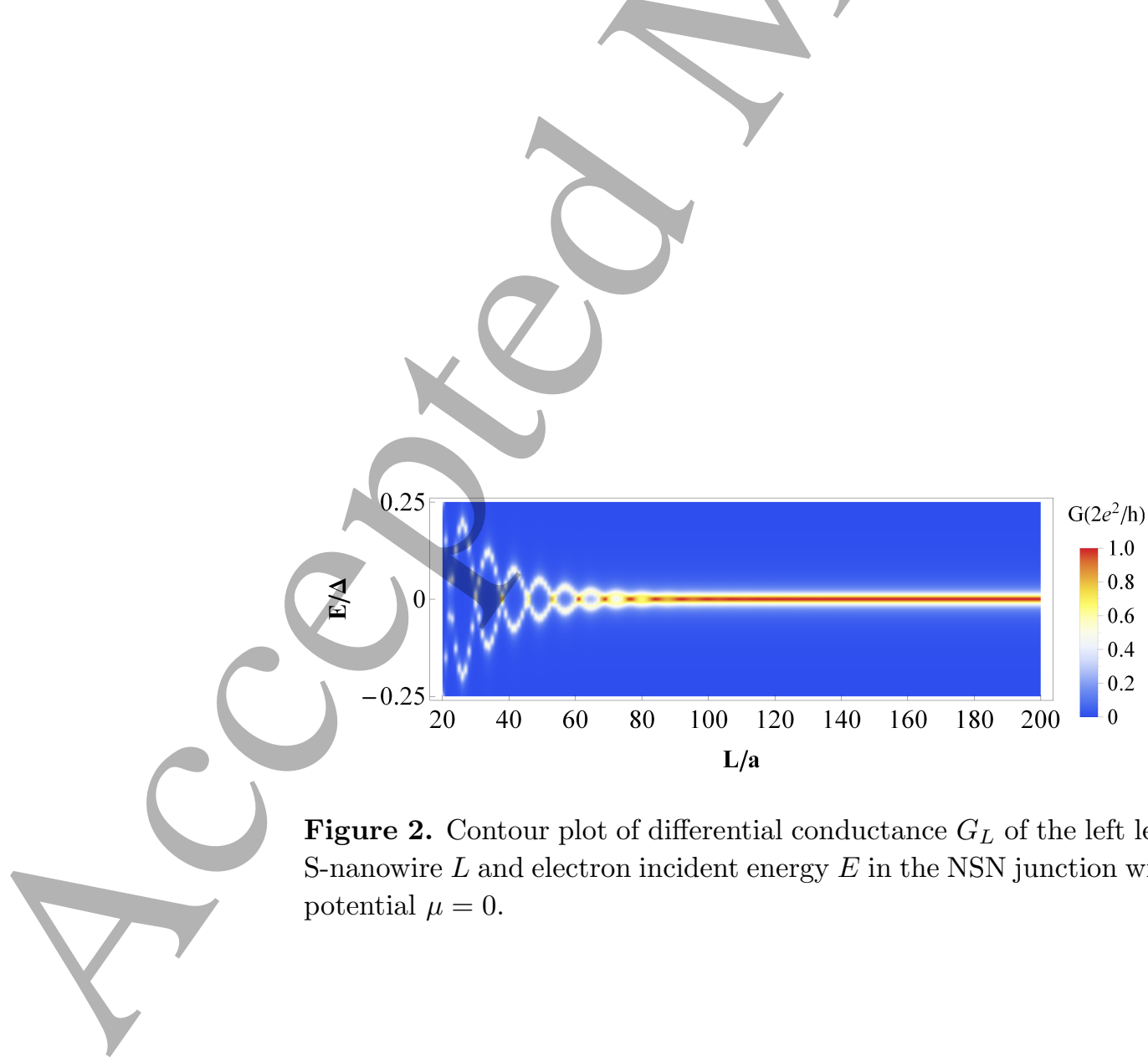

Figure 2. Contour plot of differential conductance $G_{L}$ of the left lead as the length of S-nanowire $L$ and electron incident energy $E$ in the NSN junction with a fixed chemical potential $\mu=0$. 


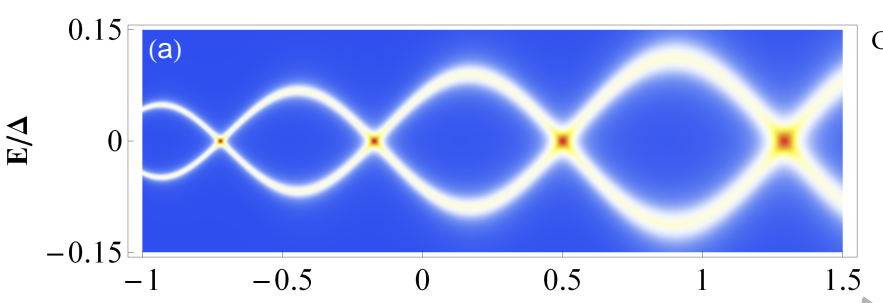

$\mathrm{G}\left(2 e^{2} / \mathrm{h}\right)$
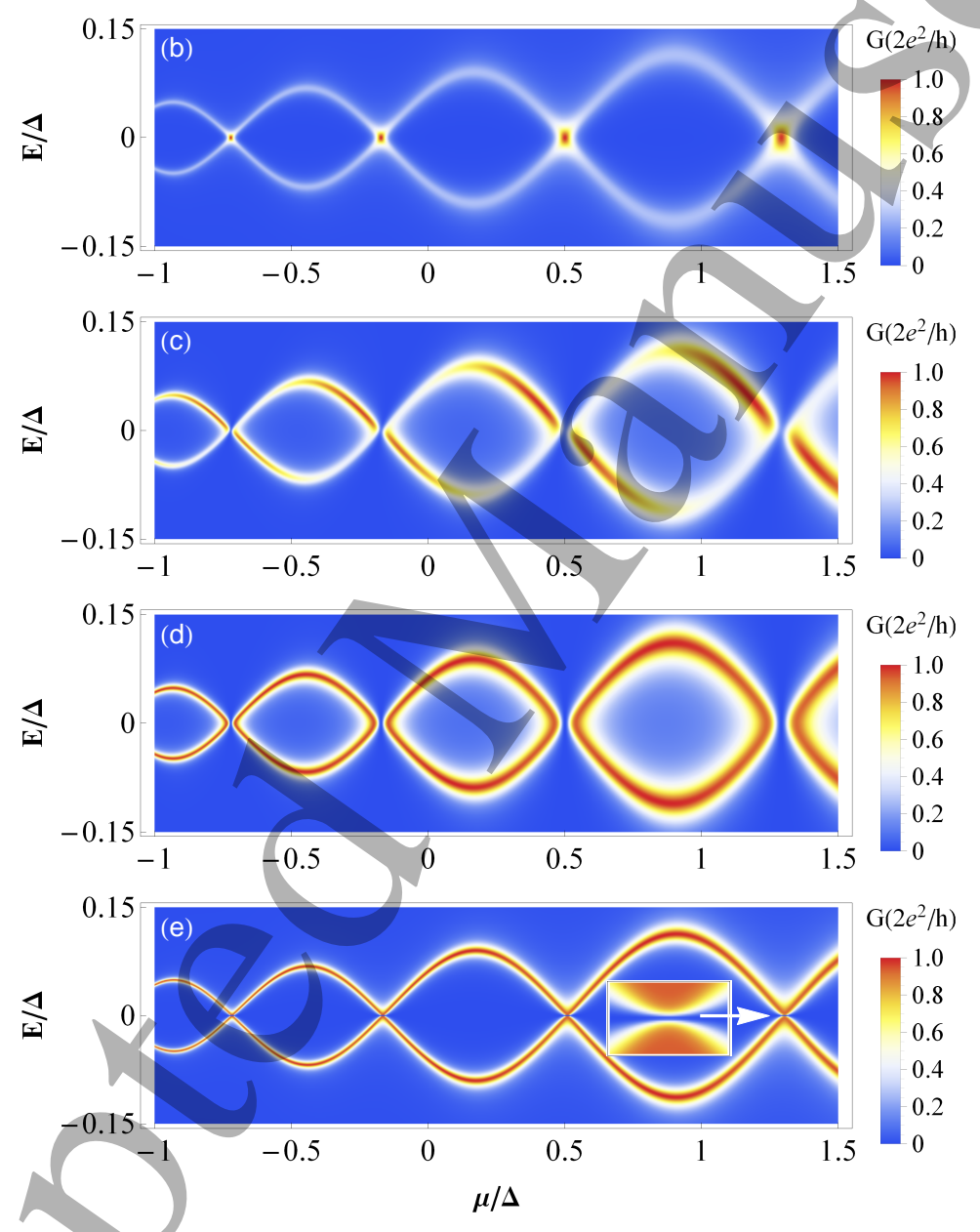

Figure 3. (a) Contour plot of differential conductance $G_{L}$ of the left lead as a function of chemical potential $\mu$ and electron incident energy $E$ in the NSN junction with a short S-nanowire. (b)-(d) correspond to its contributions (AR, CAR and ET). (e) Contour plot of differential conductance $G_{L}$ of the normal lead as a function of $\mu$ and $E$ in the NS junction with a short S-nanowire. The vanishing conductance at parity crossing points can be clearly observed in the inset. 

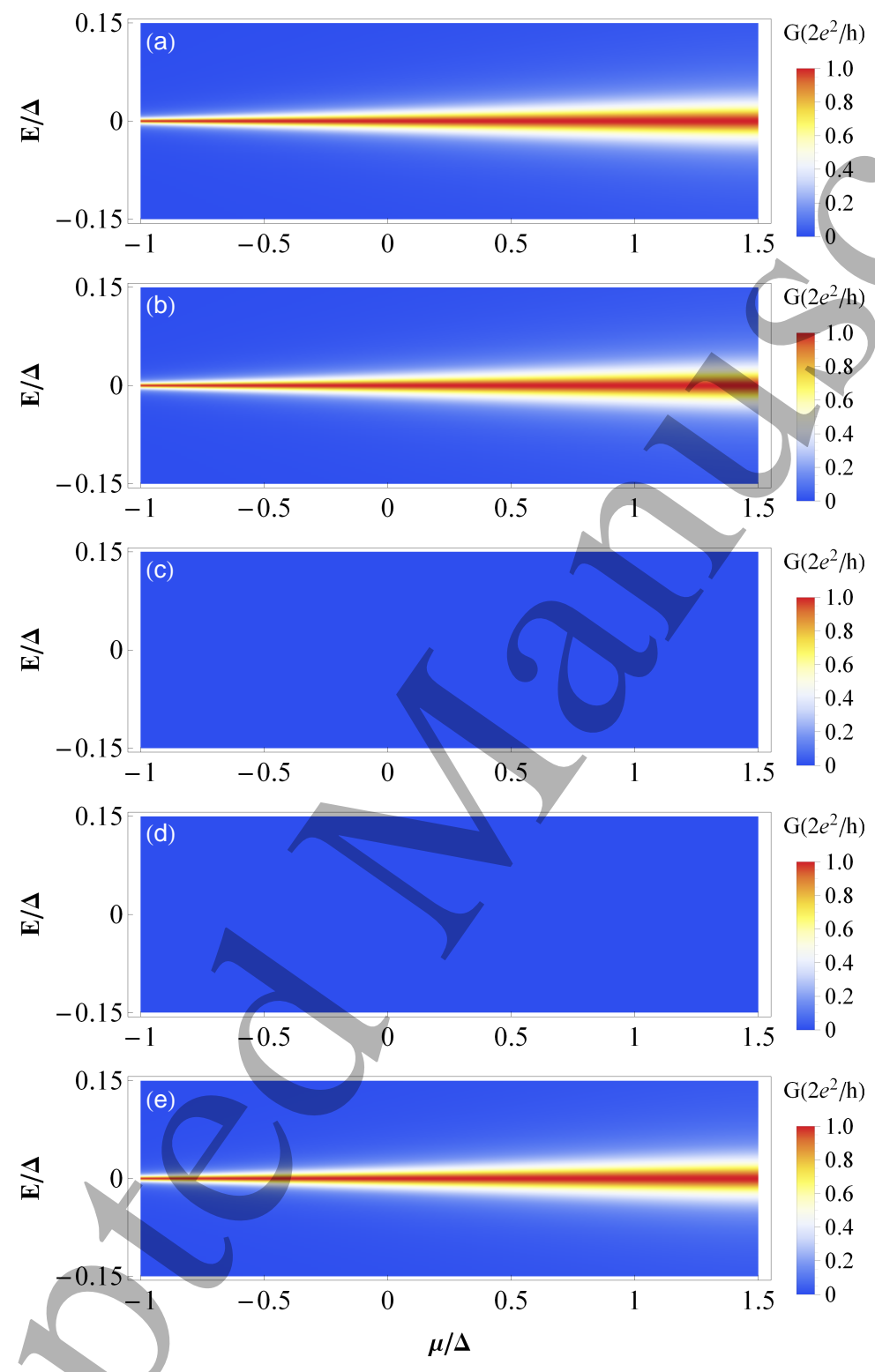

Figure 4. (a) Contour plot of differential conductance $G_{L}$ of the left lead as a function of chemical potential $\mu$ and electron incident energy $E$ in the NSN junction with a long S-nanowire. (b)-(d) correspond to its contributions (AR, CAR and ET). (e) Contour plot of differential conductance $G_{L}$ of the normal lead as a function of $\mu$ and $E$ in the NS junction with a long S-nanowire. 